\title{
Multiplex determination of murine seminal fluid cytokine profiles
}

\author{
Nadia Gopichandran, Uma V Ekbote, James J Walker, David Brooke ${ }^{1}$ and Nicolas M Orsi \\ Perinatal Research Group, Pathology and Tumour Biology, Leeds Institute of Molecular Medicine, Level 4, \\ JIF Building, St James's University Hospital, Beckett Street, Leeds LS9 7TF, UK and ${ }^{1}$ Molecular Medicine Unit, \\ Clinical Sciences Building, St James's University Hospital, Beckett Street, Leeds LS9 7TF, UK
}

Correspondence should be addressed to N M Orsi; Email: n.m.orsi@leeds.ac.uk

\begin{abstract}
Seminal fluid is known to be responsible for orchestrating mating-induced immunomodulation. Central to this process are numerous cytokines that modulate uterine leukocyte recruitment and trafficking. Despite this, a comprehensive analysis of the cytokine profile of murine seminal fluid is lacking. This study addressed this issue by using multiplex immunoassays to characterise the profile of interleukin (IL)-1 $\alpha$, IL-1 $\beta$, IL-2, IL-3, IL-4, IL-5, IL-6, IL-9, IL-10, IL-12 (p40), IL-12 (p70), IL-13, IL-17, eotaxin, granulocyte-colony stimulating factor (G-CSF), granulocyte macrophage-colony stimulating factor (GM-CSF), interferon (IFN)- $\gamma$, keratinocyte-derived chemokine (KC), monocyte chemoattractant protein (MCP)-1, macrophage inflammatory protein (MIP)-1 $\alpha$, MIP-1 $\beta$, regulated upon activation normal T-cell expressed and secreted (RANTES), and tumour necrosis factor (TNF)- $\alpha$ in fluid drawn from the seminal vesicles of single mice $(n=18)$. Their levels and ratios were compared with those found in serum. IL-1 $\alpha$, IL-1 $\beta$, IL-2, IL-5, IL-9, IL-12 (p40), IL-12 (p70), IL-13, IL-17, GM-CSF, IFN- $\gamma$, MCP-1 and TNF- $\alpha$ levels were significantly higher in serum; IL-4, G-CSF, eotaxin, KC and RANTES exhibited the opposite trend. Based on these findings, we propose a model of mating-induced immunomodulation that implicates seminal eotaxin, RANTES and MIP-1 $\alpha$ in the relocation and concentration of extravasated migrating endometrial eosinophils to the luminal epithelium. Furthermore, KC may participate in uterine neutrophil chemotaxis and activation. Eotaxin and MIP- $\alpha$, together with IL-1 $\beta$ and IL-9, may also enhance further cytokine synthesis for endometrial antigen-presenting cell recruitment for processing paternal ejaculate antigens. IL-4 and G-CSF could also minimise deleterious cell-mediated immunity and modulate IFN- $\gamma$ production, thereby supporting the establishment of pregnancy.

Reproduction (2006) 131 613-621
\end{abstract}

\section{Introduction}

The opinion that seminal plasma is not just a transport medium for the transfer of spermatozoa to the maternal tract is not recent, and its immunomodulatory properties in protecting spermatozoa, priming the uterus for pregnancy and modulating killer cell activities have long been recognized (Koch \& Ellendorff 1985, Rees et al. 1986, Saxena et al. 1988, Cardoso et al. 1993, Liang et al. 1993). In the mouse, mating induces a marked, yet transient, inflammatory response associated with endometrial leukocyte infiltration, which commonly dissipates by the time of blastocyst hatching and implantation (Robertson et al. 1997). By this stage, remaining endometrial leukocytes exhibit an immunosuppressive phenotype (Hunt et al. 1984). Studies using vasectomised males have indicated that the activation and expansion of female lymphocyte populations which occurs after mating is triggered by seminal vesicle gland constituents, and that it is independent of the presence of sperm. These lymphocytes relocate to embryo implantation sites and other mucosal tissues/lymph nodes as part of the development of maternal tolerance of the fetal allograft (Johansson et al. 2004).

This mating-induced phenomenon relies on an array of interrelated immunosuppressive mediators, including both prostaglandins, steroid binding proteins and cytokines (Tarter et al. 1986, Kelly 1995, Miao et al. 1996, Denison et al. 1999, Maccioni et al. 2001). With respect to cytokines, maternal hyporesponsiveness to paternal major histocompatibility complex (MHC) class I antigens is thought to be mediated by - at least -transforming growth factor (TGF)- $\beta 1$, regulated upon activation normal T-cell expressed and secreted (RANTES), macrophage inflammatory protein (MIP)-1 $\alpha$, MIP-1 $\beta$ and monocyte chemotactic protein (MCP)-1 (Robertson et al. 1997). Intriguingly, in man, activation of seminal plasma latent TGF- $\beta 1$ appears to be delayed until triggered by the acidic environment of 
the vagina (Nocera \& Chu 1995, Chu et al. 1996). Although alterations in the profile of some of these cytokines is not recorded in the peripheral circulation, it has been noted that murine coitus results in a fall in serum interferon (IFN)- $\gamma$ and interleukin (IL)-12 (p70) concentrations, paralleled by a rise in keratinocyte-derived chemokine (KC) and granulocyte-colony stimulating factor (G-CSF) levels in serum (Orsi et al. 2006). The immunomodulatory properties of seminal plasma have been highlighted by studies indicating that exposure to seminal fluid can: induce IL-1 $\beta$, IL-6 and leukaemia inhibitory factor (LIF) expression in human cultured endometrial epithelial cells (Gutsche et al. 2003); alter IL-8 and IL-10 release from human cervical explants, peripheral blood and monocyte cell lines in vitro (Denison et al. 1999); and elicit a rise in granulocyte macrophage-colony stimulating factor (GM-CSF) in murine uterine luminal epithelial cells (Robertson et al. 1996a). The alterations in cytokine ratios (e.g. IL-10:IL-12) activated by semen deposition are believed to exercise a key inhibitory control over vital immune defences in the lower genital tract, with ablation of cell-mediated responses and immunosurveillance (Kelly et al. 1997). A comprehensive review of the role of seminal plasma and male factor signalling in the maternal tract has recently been conducted by Robertson (2005).

In man, there has been extensive interest in seminal plasma cytokine profile, which has been characterised for IL-1 $\alpha / \beta$, IL-2, IL-4, IL-6, IL-8, IL-10, IL-11, IL-12, tumour necrosis factor (TNF)- $\alpha / \beta$, IFN $-\alpha / \gamma$, TGF- $\beta 1$, RANTES, CSF-1, G-CSF, macrophage-CSF, stem cell factor, monocyte chemotactic and activating factor, macrophage migration inhibitory factor and vascular endothelial growth factor as well as an array of their soluble receptors (Naz \& Stanley 1995, Shimoya et al. 1995, Frenette et al. 1998, Fujisawa et al. 1998a,b, Matalliotakis et al. 1998a,b, 2002, Omu et al. 1998, Huleihel et al. 1999, Naz \& Leslie 2000, Maegawa et al. 2002, Gutsche et al. 2003, Paulis et al. 2003, Basu et al. 2004). However, these studies have been limited in the array of cytokines investigated in individual patients, and their focus has principally been on the association of these inflammatory mediators with a variety of disorders resulting in male factor infertility and/or characterised by inflammatory processes. By contrast, surprisingly little is known about both the identity and the concentration of cytokines in murine seminal fluid, principally due to the small volume of analysable sample.

The present study therefore aimed to: (a) establish the physiological profiles of cytokines in murine seminal fluid from single animals, and their relative ratios, and (b) compare these profiles with those measured in serum. Fluidphase multiplex immunoassays were used to overcome the difficulties associated with multiple analyses on small sample volumes from individual animals (Orsi et al. 2006). Seminal vesicle fluid was used since it was straightforward to collect, and because it is understood to contain the immunomodulatory factors that act on the maternal tract. There is no evidence to implicate the accessory glands in this process as seminal vesicle-deficient males are unable to elicit the typical response exhibited by their entire counterparts in terms of GM-CSF rise in the female tract (Robertson et al. 1996a).

\section{Materials and Methods}

CD1 virgin male mice (10-12 weeks old; $n=18)$ were killed by cervical dislocation in compliance with the Animals (Scientific Procedures) Act, 1986. Seminal vesicles were dissected while avoiding exposure to fluid from the coagulating gland, and collection was carried out by gentle massaging. Since difficulties were anticipated in analysing pure seminal fluid due its density, samples were immediately diluted with $200 \mu$ l sterile PBS with $0.5 \%$ BSA (as recommended by A Liversage, BioRad Laboratories) and thoroughly vortexed for $45 \mathrm{~s}$. This dilution was taken into account for each sample when determining sample-specific cytokine profiles. In parallel, serum was isolated from blood obtained by post-mortem cardiac puncture. Blood and seminal fluid samples were all centrifuged at 9000 r.p.m. for 3 min on a microcentrifuge (Micro Centaur, MSE Scientific, Loughborough, UK). The supernatant was frozen at $-80^{\circ} \mathrm{C}$ until analysed simultaneously for the following 23 cytokines: IL-1 $\alpha$, IL-1 $\beta$, IL-2, IL-3, IL-4, IL-5, IL-6, IL-9, IL-10, IL-12 (p40), IL-12 (p70), IL-13, IL-17, eotaxin, G-CSF, GM-CSF, IFN- $\gamma$, KC, MCP-1, MIP- $1 \alpha$, MIP- $1 \beta$, RANTES and TNF- $\alpha$. This was achieved by 23-plex fluid-phase immunoassay using custom kits (BioRad Laboratories) run on a Luminex100 cytometer (Luminex Corporation, Austin, TX, USA), equipped with StarStation software (Applied Cytometry Systems, Dinnington, UK) (Vignali 2000, Powell et al. 2004). Serum diluent was used in all cases to avoid false positive/ negatives and dilution adjusted to $1: 1$ in order to maximise sensitivity to baseline levels (Orsi et al. 2006). All samples were analysed in duplicate. Cytokine levels were expressed in picogrammes per millilitre. Biofluid cytokine ratios were determined to highlight the interrelationships between cytokines. In rare cases where no analyte was detected for a specific cytokine, ratios could not be performed for that individual animal. All data were expressed as means \pm S.E.M. Data distributions were assessed by Anderson-Darling tests and significant differences between groups were determined using Student's $t$ tests or Mann-Whitney $U$ tests, as appropriate.

\section{Results}

\section{Serum and seminal fluid cytokine profiles}

The profiles of all cytokines analysed were detected in both biofluids, with the exception of serum IL-3, which was below the sensitivity level of the assay (Fig. 1). The lowest detectable serum cytokine concentrations were noted for IL-4 and RANTES (both $<1 \mathrm{pg} / \mathrm{ml}$ ); low levels were recorded for IL-2, IL-5, IL-6, IL-10 and MIP-1 $\beta$ (5-20 pg/ml range); intermediate levels for IL-1 $\alpha$, IL-12 

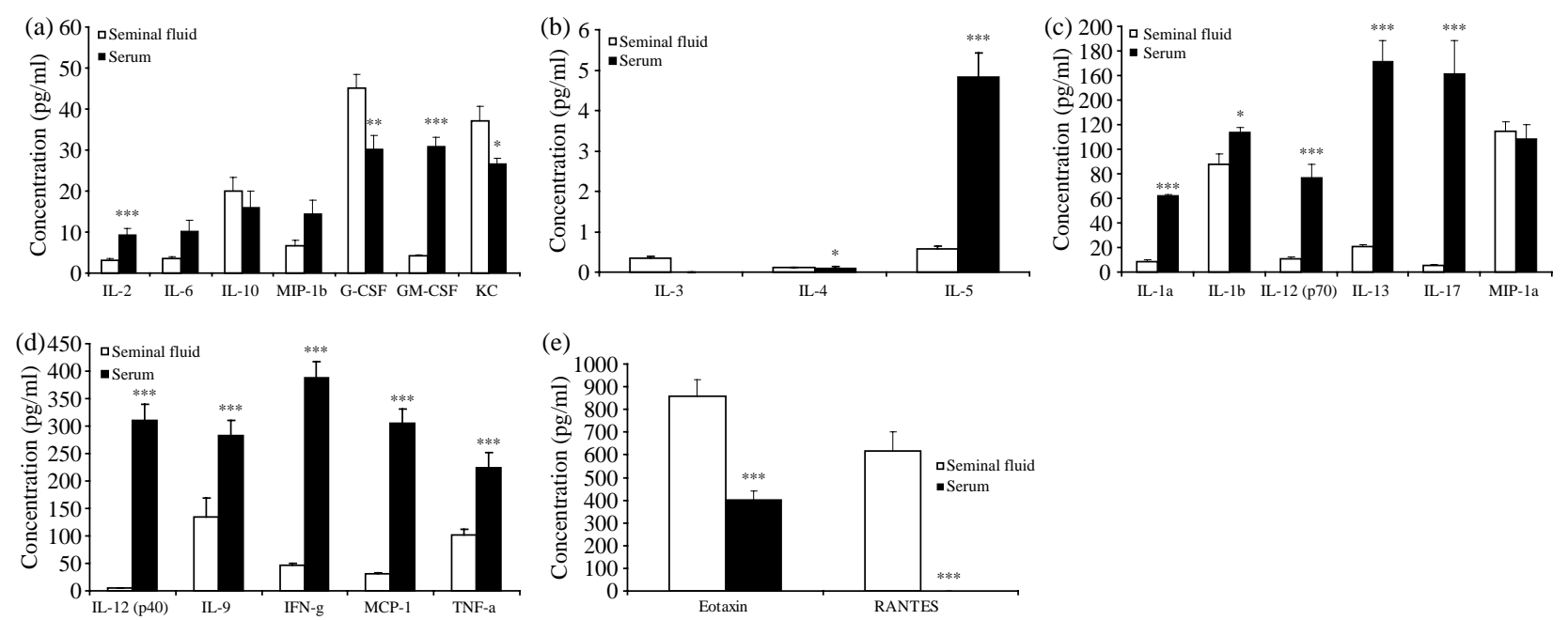

Figure 1 Serum and seminal fluid concentrations in male CD1 mice: (a) IL-2, IL-6, IL-10, MIP-1 $\beta$, G-CSF, GM-CSF and KC; (b) IL-3, IL-4 and IL-5; (c) IL-1 $\alpha$, IL-1 $\beta$, IL-12 (p70), IL-13, IL-17 and MIP-1 $\alpha$; (d) IL-12 (p40), IL-9, IFN- $\gamma$, MCP-1 and TNF- $\alpha$; (e) eotaxin and RANTES.

(p70), G-CSF, GM-CSF and KC (25-80 pg/ml); and higher levels were identified for IL-1 $\beta$, IL-13, IL-17, MIP-1 $\alpha$ and TNF- $\alpha(100-250 \mathrm{pg} / \mathrm{ml})$. The most prevalent cytokines in serum were IL-9, IL-12 (p40), eotaxin, MCP-1 and IFN- $\gamma$ $(250-400 \mathrm{pg} / \mathrm{ml})$.

The lowest levels detected in seminal fluid were for IL-3, IL-4 and IL-5 (all $<1 \mathrm{pg} / \mathrm{ml}$ ). Relatively low concentrations of IL-1 $\alpha$, IL-2, IL-6, IL-12 (p40), IL-12 (p70), IL-17, MIP-1 $\beta$ and GM-CSF $(<12 \mathrm{pg} / \mathrm{ml})$ were detected; IL-1 $\beta$, IL-9, IL-10, IL-13, G-CSF, MCP-1, MIP- $1 \alpha, \mathrm{KC}$, IFN- $\gamma$ and TNF- $\alpha$ were intermediate $(20-150 \mathrm{pg} / \mathrm{ml}$ range), while by far the most prevalent cytokines in seminal fluid were RANTES and eotaxin (500-900 pg/ml range).

Cytokine levels in serum and seminal fluid were markedly different: the majority were significantly higher in serum, as indicated for IL-1 $\alpha$, IL-1 $\beta$, IL-2, IL-5, IL-9, IL-12 (p40), IL-12 (p70), IL-13, IL-17, GM-CSF, IFN- $\gamma$, MCP-1 and TNF- $\alpha$. By contrast, only IL-4, G-CSF, KC, eotaxin and RANTES levels were significantly higher in seminal fluid. No significant differences in IL-6, IL-10 and MIP-1 $\alpha$ and MIP-1 $\beta$ were detected between seminal plasma and serum.

\section{Comparisons of serum and seminal fluid cytokine ratios}

With very few exceptions, the ratios between the different cytokines were strikingly different between serum and seminal fluid (Tables 1 and 2). Because IL-3 serum levels were not quantified, serum ratios and seminal fluid comparisons are not available for this cytokine. Likewise, the single detectable serum RANTES sample prevented meaningful ratio comparisons between serum and seminal fluid.

\section{Discussion}

To the best of our knowledge, this study is the first study to characterise extensively the profile of 23 cytokines (many of these novel) in the serum and seminal fluid of single mice using multiplex immunoassays. The serum cytokine concentrations reported here are comparable, in some respects, to those identified in C57/Bl6 female mice during the oestrous cycle and in early pregnancy using multiplex immunoassays (Orsi et al. 2006). Noteworthy exceptions in CD1 males were: much higher MIP-1 $\alpha$ and IFN- $\gamma$ levels; elevated IL- $1 \alpha$, IL-17, TNF- $\alpha$ and GM-CSF concentrations; and lower IL-6, IL-10, G-CSF, RANTES and IL-12 (p40) profiles. Whether these effects are attributable to animal strain, gender, or both, remains unclear.

It is well documented that factors within seminal plasma act to enhance the receptivity of the maternal tract and immune system for the subsequent pregnancy. In particular, immunomodulatory moieties within murine seminal plasma elicit the relocation of antigen-presenting cells to the uterus where they participate in the presentation of paternal ejaculate antigens to activate lymphocytes (Robertson et al. 2003, O'Leary et al. 2004). Furthermore, they orchestrate leukocyte trafficking and activate uterine epithelial cytokine expression, such as GM-CSF and IL-6 (Robertson et al. 1992, Tremellen et al. 1998). Several of the cytokines found in murine seminal plasma have been credited with immunosuppressive activity, including IL-1, IL-6 and IL-8 (Kelly 1999, Robertson \& Sharkey 2001).

Certain cytokine profiles were modest in both serum and semen - as noted for IL-2, IL-3, IL-4, IL-5 and IL-6. Although this may reflect their lesser importance in the early cytokine signalling induced by seminal plasma, these interleukins may nonetheless be involved in subsequent events associated with uterine priming and the establishment of pregnancy. In this respect, seminal plasma has been shown to elicit a 200-fold increase in IL-6 levels in oestrogen-primed uterine epithelial cells (Robertson et al. 1997), while the circulatory level of all these cytokines has been shown to increase significantly in the latter half of gestation (Orsi et al. 2006). 


\begin{tabular}{|c|c|c|c|c|c|c|c|c|c|c|c|c|}
\hline & IL-1 $\beta$ & IL-2 & IL-3 & IL-4 & IL-5 & IL-6 & IL-9 & IL-10 & IL-12 (p40) & IL-12 (p70) & IL-13 & IL-17 \\
\hline IL-1 $\alpha$ & $1.83 \pm 0.06$ & $0.15 \pm 0.02$ & * & $0.01 \pm 0.01$ & $0.08 \pm 0.01$ & $0.17 \pm 0.04$ & $4.54 \pm 0.45$ & $0.30 \pm 0.06$ & $4.96 \pm 0.48$ & $1.29 \pm 0.16$ & $2.91 \pm 0.22$ & $2.56 \pm 0.42$ \\
\hline IL-1 $\beta$ & $*$ & $0.08 \pm 0.01$ & * & $0.01 \pm 0.01$ & $0.04 \pm 0.01$ & $0.09 \pm 0.02$ & $2.55 \pm 0.31$ & $0.16 \pm 0.03$ & $2.73 \pm 0.25$ & $0.71 \pm 0.09$ & $1.58 \pm 0.12$ & $1.39 \pm 0.22$ \\
\hline IL-2 & * & $*$ & * & $0.07 \pm 0.04$ & $5.79 \pm 3.62$ & $1.81 \pm 0.46$ & $229.46 \pm 135.12$ & $10.71 \pm 6.27$ & $328.02 \pm 176.45$ & $56.91 \pm 39.92$ & $227.75 \pm 186.40$ & $121.79 \pm 65.55$ \\
\hline IL-3 & * & * & * & $*$ & $*$ & $*$ & $*$ & $*$ & $*$ & $*$ & $*$ & $*$ \\
\hline IL-4 & * & * & * & * & $78.88 \pm 13.16$ & $126.46 \pm 22.71$ & $4906.65 \pm 890.35$ & $216.93 \pm 22.09$ & $5425.36 \pm 880.93$ & $1318.67 \pm 202.10$ & $3012.91 \pm 486.63$ & $2427.36 \pm 337.04$ \\
\hline IL-5 & * & * & * & * & $*$ & $2.15 \pm 0.57$ & $69.38 \pm 10.07$ & $4.24 \pm 0.97$ & $80.01 \pm 12.08$ & $20.53 \pm 4.29$ & $39.90 \pm 2.96$ & $40.68 \pm 8.20$ \\
\hline IL-6 & * & * & * & * & * & $*$ & $65.97 \pm 19.99$ & $2.35 \pm 0.79$ & $80.12 \pm 24.34$ & $11.42 \pm 2.19$ & $38.19 \pm 13.46$ & $25.49 \pm 4.84$ \\
\hline IL-9 & * & * & * & * & * & * & $*$ & $0.07 \pm 0.02$ & $1.63 \pm 0.41$ & $0.30 \pm 0.04$ & $0.66 \pm 0.07$ & $0.72 \pm 0.13$ \\
\hline IL-10 & * & * & * & * & * & * & * & $*$ & $25.33 \pm 4.46$ & $6.35 \pm 0.94$ & $15.17 \pm 2.86$ & $12.41 \pm 1.52$ \\
\hline $\begin{array}{l}\text { IL-12 } \\
\text { (p40) }\end{array}$ & * & * & $*$ & * & * & $*$ & $*$ & $*$ & $*$ & $0.29 \pm 0.04$ & $0.75 \pm 0.17$ & $0.55 \pm 0.09$ \\
\hline $\begin{array}{l}\text { IL-12 } \\
\text { (p70) }\end{array}$ & $*$ & * & $*$ & * & * & * & $*$ & * & $*$ & * & $2.96 \pm 0.52$ & $2.05 \pm 0.16$ \\
\hline IL-13 & * & * & * & * & * & * & * & * & * & * & * & $1.06 \pm 0.20$ \\
\hline
\end{tabular}

Table 2 Serum cytokine ratios (statistical comparisons with seminal fluid are indicated in Table 4).

\begin{tabular}{|c|c|c|c|c|c|c|c|c|c|c|}
\hline & Eotaxin & G-CSF & GM-CSF & IFN- $\gamma$ & КC & MCP-1 & MIP-1 $\alpha$ & MIP-1及 & RANTES & TNF- $\alpha$ \\
\hline IL-1 $\alpha$ & $6.77 \pm 0.46$ & $0.48 \pm 0.05$ & $0.49 \pm 0.04$ & $6.23 \pm 0.46$ & $0.43 \pm 0.02$ & $4.91 \pm 0.42$ & $1.73 \pm 0.18$ & $0.26 \pm 0.05$ & 0.14 & $3.58 \pm 0.42$ \\
\hline IL-1 $\beta$ & $3.68 \pm 0.24$ & $0.27 \pm 0.03$ & $0.27 \pm 0.02$ & $3.41 \pm 0.25$ & $0.24 \pm 0.01$ & $2.70 \pm 0.23$ & $0.95 \pm 0.09$ & $0.14 \pm 0.03$ & 0.08 & $1.97 \pm 0.23$ \\
\hline IL-2 & $45.25 \pm 6.29$ & $47.62 \pm 27.69$ & $30.15 \pm 18.07$ & $574.95 \pm 407.74$ & $53.71 \pm 33.64$ & $449.23 \pm 299.08$ & $160.58 \pm 96.03$ & $4.63 \pm 3.05$ & 1.22 & $157.76 \pm 89.14$ \\
\hline IL-3 & $*$ & $*$ & $*$ & $*$ & $*$ & $*$ & $*$ & $*$ & $*$ & $*$ \\
\hline IL-4 & $7232.90 \pm 1295.27$ & $529.32 \pm 98.73$ & $510.04 \pm 76.57$ & $6792.30 \pm 1169.26$ & $520.20 \pm 94.38$ & $5485.62 \pm 960.66$ & $1740.93 \pm 215.42$ & $202.46 \pm 30.23$ & 17.76 & $3480.01 \pm 447.94$ \\
\hline IL-5 & $95.18 \pm 6.77$ & $8.20 \pm 1.41$ & $7.18 \pm 0.59$ & $91.65 \pm 7.76$ & $7.56 \pm 1.32$ & $76.16 \pm 9.14$ & $28.61 \pm 4.36$ & $3.48 \pm 0.74$ & 2.30 & $53.59 \pm 7.62$ \\
\hline IL-6 & $75.75 \pm 20.58$ & $9.18 \pm 3.61$ & $6.88 \pm 1.88$ & $87.33 \pm 240.32$ & $10.36 \pm 5.04$ & $72.61 \pm 21.71$ & $31.31 \pm 13.45$ & $2.01 \pm 0.78$ & 0.25 & $41.10 \pm 10.21$ \\
\hline IL-9 & $1.57 \pm 0.16$ & $0.17 \pm 0.06$ & $0.13 \pm 0.01$ & $1.65 \pm 0.22$ & $0.18 \pm 0.08$ & $1.37 \pm 0.25$ & $0.67 \pm 0.23$ & $0.06 \pm 0.01$ & 0.04 & $0.88 \pm 0.11$ \\
\hline IL-10 & $33.38 \pm 5.01$ & $2.80 \pm 0.87$ & $2.58 \pm 0.43$ & $34.71 \pm 6.67$ & $2.39 \pm 0.58$ & $28.97 \pm 6.55$ & $9.04 \pm 1.24$ & $0.96 \pm 0.13$ & 0.15 & $18.35 \pm 2.67$ \\
\hline IL-12 (p40) & $1.59 \pm 0.22$ & $0.11 \pm 0.01$ & $0.12 \pm 0.02$ & $1.61 \pm 0.34$ & $0.11 \pm 0.02$ & $1.41 \pm 0.43$ & $0.40 \pm 0.06$ & $0.05 \pm 0.01$ & 0.03 & $0.85 \pm 0.18$ \\
\hline IL-12 (p70) & $6.38 \pm 0.74$ & $0.46 \pm 0.07$ & $0.49 \pm 0.07$ & $6.34 \pm 0.88$ & $0.43 \pm 0.07$ & $5.07 \pm 0.78$ & $1.57 \pm 0.19$ & $0.18 \pm 0.02$ & 0.12 & $3.23 \pm 0.29$ \\
\hline IL-13 & $2.46 \pm 0.16$ & $0.19 \pm 0.03$ & $0.19 \pm 0.01$ & $2.30 \pm 0.08$ & $0.16 \pm 0.02$ & $1.82 \pm 0.10$ & $0.68 \pm 0.10$ & $0.09 \pm 0.02$ & 0.06 & $1.40 \pm 0.15$ \\
\hline IL-17 & $3.41 \pm 0.45$ & $0.28 \pm 0.05$ & $0.26 \pm 0.03$ & $3.38 \pm 0.46$ & $0.28 \pm 0.06$ & $2.75 \pm 0.39$ & $0.88 \pm 0.14$ & $0.09 \pm 0.01$ & 0.05 & $1.62 \pm 0.17$ \\
\hline Eotaxin & $*$ & $0.08 \pm 0.01$ & $0.08 \pm 0.01$ & $0.99 \pm 0.06$ & $0.07 \pm 0.01$ & $0.80 \pm 0.08$ & $0.27 \pm 0.03$ & $0.04 \pm 0.01$ & 0.02 & $0.57 \pm 0.05$ \\
\hline G-CSF & * & * & $1.20 \pm 0.16$ & $15.53 \pm 2.23$ & $1.09 \pm 0.14$ & $12.93 \pm 2.54$ & $3.98 \pm 0.47$ & $0.55 \pm 0.09$ & 0.17 & $8.65 \pm 1.26$ \\
\hline GM-CSF & * & * & * & $12.93 \pm 0.66$ & $1.11 \pm 0.25$ & $10.51 \pm 0.76$ & $4.13 \pm 0.71$ & $0.46 \pm 0.08$ & 0.27 & $7.08 \pm 0.58$ \\
\hline $\mathrm{IFN}-\gamma$ & * & * & * & $*$ & $0.08 \pm 0.02$ & $0.81 \pm 0.04$ & $0.33 \pm 0.05$ & $0.04 \pm 0.01$ & 0.03 & $0.57 \pm 0.05$ \\
\hline $\mathrm{KC}$ & * & * & * & * & $*$ & $11.85 \pm 1.09$ & $4.41 \pm 0.59$ & $0.65 \pm 0.14$ & 0.42 & $8.81 \pm 1.16$ \\
\hline MCP-1 & * & * & * & * & * & $*$ & $0.40 \pm 0.05$ & $0.05 \pm 0.01$ & 0.04 & $0.74 \pm 0.08$ \\
\hline MIP-1 $\alpha$ & * & * & * & * & * & * & $*$ & $0.17 \pm 0.05$ & 0.07 & $2.60 \pm 0.55$ \\
\hline MIP-1 $\beta$ & * & * & * & * & * & * & * & $*$ & 0.46 & $29.84 \pm 7.71$ \\
\hline RANTES & * & * & * & * & * & * & * & * & $*$ & 25.09 \\
\hline
\end{tabular}


By contrast, other cytokines were present at comparatively high concentrations in both serum and semen; namely eotaxin, MIP- $\alpha$, IL-1 $\beta$ and IL-9. However, while eotaxin and MIP- $1 \alpha$ consistently displayed proportionally higher ratios in semen than serum, only eotaxin levels were significantly higher in seminal plasma. In this respect, at oestrus, IL-5 recruits circulatory eosinophils into the uterine stroma, while the presence of ovarian steroids stimulates the synthesis of endometrial eotaxin, RANTES and MIP-1 $\alpha$ (Robertson et al. 2000) (Fig. 2). In turn, these elicit the extravasation and movement of eosinophils through the stroma to the epithelium (Robertson et al. 2000, Kayisli et al. 2002). In the presence of semen their abundance and proximity to the luminal surface peaks. Speculatively, high seminal eotaxin and RANTES levels may further operate directly on these eosinophils as they express - at least - an eotaxin-specific receptor which may regulate their selective recruitment (Mould et al. 1997). Unlike in man, murine coitus results in the deposition of semen directly into the uterus where, as allogenic material, it triggers an acute inflammatory response (Wood et al. 1997). Thus, seminal fluid constituents - potentially including eotaxin, MIP- $\alpha$, IL- $1 \beta$ and IL9 - initiate a surge in the synthesis of other cytokines (e.g. IL-6 and GM-CSF) which subsequently elicits the recruitment and activation of antigen-presenting cells into the endometrial stroma where they engulf and process paternal ejaculate antigens. Some of these may be eosinophils, which have recognised phagocytic and antigen-processing functions (Strath \& Sanderson 1985, Xie et al. 2005). Intriguingly, the present data do not support a role for seminal IL-13 in eosinophil recruitment (Wills-Karp 1999). These then relocate to the para-aortic lymph nodes which drain the uterus (Robertson et al. 2003). In parallel, potentially in response to the array of cytokines described in this study, granulocytes and macrophages accumulate in the uterus to clear sperm and seminal debris (Wood et al. 1997). Thus, these cytokines, together with seminal TGF- $\beta 1$, may contribute to the localised inflammatory response which (a) acts to prime the uterus for subsequent embryo paracrine interactions, implantation and fetal growth, and (b) prepares the maternal immune system for exposure to paternal antigens, thus ensuring the successful establishment of pregnancy.

In addition, seminal fluid was also characterised by higher levels of IL-4 and G-CSF compared with serum, suggesting that these too may have a role, as yet undefined, in mating-induced immunomodulation. In man, IL-4 is thought to be necessary for the establishment and maintenance of pregnancy, by avoiding the harmful effects of cell-mediated immunity in the vicinity of putative embryo implantation sites and at the feto-maternal

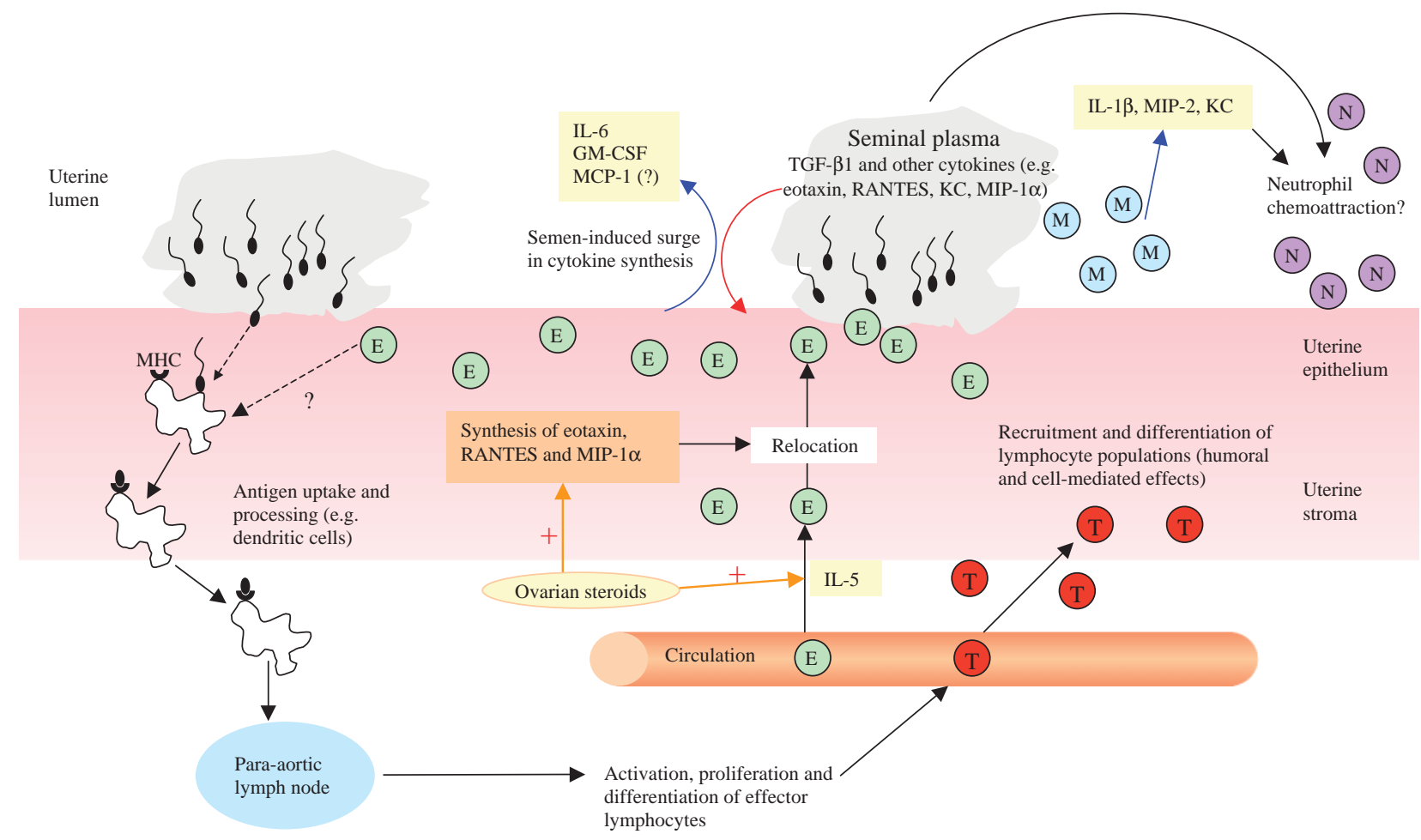

Figure 2 Seminal plasma-induced immunomodulation. Seminal cytokines attract eosinophils (E) to the uterine luminal surface following their chemotactic relocation from the circulation in response to endometrial cytokines. Seminal cytokines may also contribute to neutrophil $(\mathrm{N})$ chemoattraction while seminal antigens are processed by dendritic cells. These relocate to the para-aortic lymph nodes and induce the activation, proliferation and differentiation of T-cell (T) subsets. These subsequently relocate into the uterine endometrium by the time of embryo implantation, by which time macrophages $(M)$ have cleared residual seminal debris. 
interface (Lin et al. 1993, Piccinni 2005). Similarly, G-CSF, which participates in subsequent placental granulocytosis, can modulate the production of deleterious levels of IFN- $\gamma$ (which we report to be comparatively low in seminal fluid), which is known to impair human trophoblast cell growth and function in vitro and to cause abortion in mice (Sugita et al. 2003; reviewed by Daher et al. 2004).

Seminal fluid and serum cytokines were expressed as ratios in order to further highlight their differences (Tables 3 and 4). Expressing cytokine profiles in this way is useful as these glycoproteins operate in a network system that involves numerous inhibitory and synergistic interactions. The evident ratio differences found in serum and seminal fluid probably reflect their differing specific functions. In particular, the most pronounced differences in serum and seminal ratios were noted for KC, RANTES (proportionally higher in semen), IL-17 and IL-12 (p40) (which showed the inverse relationship). The relatively high proportion (and indeed overall levels) of KC to other cytokines in seminal fluid compared with serum may belie its function in the chemotaxis and activation of neutrophils associated with murine coitus (Knudsen et al. 2002). Indeed, it has been reported that the most noteworthy effect of mating seminal vesicle-deficient stud mice was a complete absence of neutrophils in the uterine luminal cavity (Robertson et al. 1996b). We propose that this effect may be attributable, at least in part, to an absence of RANTES, eotaxin, G-CSF and KC in these animals, which would normally orchestrate neutrophil relocation to the site of seminal deposition. With respect to RANTES, in addition to its role in endometrial eosinophil recruitment, high levels may participate in immunomodulation of antigenicity of sperm cells in the male genital tract prior to ejaculation, and perhaps also in the female tract following mating (Naz \& Leslie 2000, Kayisli et al. 2002). By contrast, the proportionally lower seminal fluid IL-17 levels (reflected in the ratios) may be accounted for by its adverse pro-inflammatory properties which - while participating in the induction of IL-1 $\beta$, IL-6, IL-8, G-CSF and MCP-1 - are actually associated with transplant rejection and other disorders of inflammatory origin (Fossiez et al. 1998, Zhang et al. 2005). Thus, the low levels of IL-17 we have identified in semen may be conducive to developing the immunopermissive environment required for tolerance of the conceptus upon its entry into the uterus. The exact meaning of the strikingly lower IL-12 (p40) ratios (and levels) in semen is likely to be very complex. As well as being a subunit of other cytokines (e.g. IL12 (p70) and IL-23), it acts as an antagonist of the p70 heterodimer. However, high levels of IL-12 (p40) have been associated with an acceleration of allograft rejection in vivo (Sun et al. 2004), thereby suggesting that low levels of IL-12 (p40) may favour successful implantation as outlined for IL-17.

All cytokine profiles were determined on a homogeneous population of virgin CD1 male mice. Although these values are no doubt representative of real differences

Reproduction (2006) 131 613-621

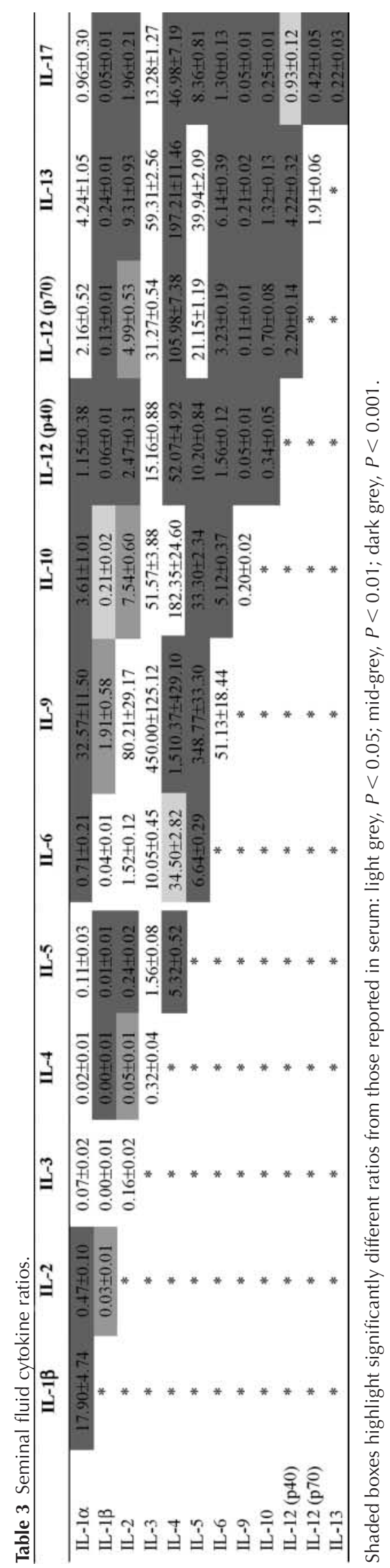

www.reproduction-online.org 
Table 4 Seminal fluid cytokine ratios.

\begin{tabular}{|c|c|c|c|c|c|c|c|c|c|c|}
\hline & Eotaxin & G-CSF & GM-CSF & IFN- $\gamma$ & KC & MCP-1 & MIP-1 $\alpha$ & MIP- $1 \beta$ & RANTES & TNF- $\alpha$ \\
\hline $\mathrm{IL}-1 \alpha$ & $184.56 \pm 52.84$ & $9.35 \pm 1.98$ & $0.87 \pm 0.24$ & $9.76 \pm 2.61$ & $8.10 \pm 2.42$ & $6.06 \pm 1.42$ & $24.57 \pm 6.36$ & $1.33 \pm 0.42$ & $163.37 \pm 50.39$ & $20.73 \pm 5.22$ \\
\hline $\mathbb{L}-1 \beta$ & $10.43 \pm 0.65$ & $0.56 \pm 0.03$ & $0.05 \pm 0.01$ & $0.56 \pm 0.03$ & $0.44 \pm 0.04$ & $0.35 \pm 0.01$ & $1.39 \pm 0.06$ & $0.07 \pm 0.01$ & $8.19 \pm 1.11$ & $1.21 \pm 0.03$ \\
\hline$\amalg-2$ & $404.90 \pm 49.24$ & $22.58+3.36$ & $1.94 \pm 0.22$ & $21.71+2.50$ & $17.63 \pm 2.23$ & $13.90 \pm 158$ & $56.26 \pm 7.20$ & $2.63 \pm 0.37$ & $356.10 \pm 70.95$ & $47.93 \pm 5.50$ \\
\hline $\mathrm{IL}-3$ & $2508.71 \pm 140.93$ & $134.82 \pm 7.99$ & $12.06 \pm 0.31$ & $136.70 \pm 6.65$ & $111.04 \pm 11.04$ & $87.23 \pm 3.75$ & $342.49 \pm 16.71$ & $17.42 \pm 2.13$ & $2065.90 \pm 316.28$ & $297.46 \pm 7.99$ \\
\hline IL-4 & $8420.08 \pm 549.71$ & $449.53 \pm 31.09$ & $40.76 \pm 2.93$ & $451.42+27.96$ & $356.82 \pm 29.40$ & $291.80 \pm 18.52$ & $1133.61 \pm 73.40$ & $61.87 \pm 9.76$ & $6690.93 \pm 975.76$ & $1001.66 \pm 67.80$ \\
\hline IL-5 & $1741.26 \pm 142.13$ & $93.15 \pm 7.41$ & $8.04 \pm 0.41$ & $92.76 \pm 6.25$ & $72.96 \pm 6.44$ & $58.69 \pm 2.95$ & $232.62+17.12$ & $10.95 \pm 1.42$ & $1389.11 \pm 217.73$ & $202.42 \pm 12.94$ \\
\hline IL-6 & $264.75 \pm 20.20$ & $14.27 \pm 1.20$ & $1.23 \pm 0.07$ & $14.09 \pm 0.95$ & $11.33 \pm 1.09$ & $9.08 \pm 0.63$ & $35.84 \pm 2.95$ & $1.67 \pm 0.22$ & $212.71+31.83$ & $30.91 \pm 2.03$ \\
\hline IL-9 & $8.59 \pm 0.85$ & $0.49 \pm 0.06$ & $0.04 \pm 0.01$ & $0.49 \pm 0.05$ & $0.39 \pm 0.04$ & $0.32 \pm 0.03$ & $1.21 \pm 0.14$ & $0.06 \pm 0.01$ & $6.53 \pm 1.25$ & $1.08 \pm 0.12$ \\
\hline IL-10 & $59.16 \pm 8.21$ & $3.14 \pm 0.39$ & $0.27 \pm 0.03$ & $3.07 \pm 0.35$ & $2.49 \pm 0.34$ & $1.93 \pm 0.17$ & $7.90 \pm 1.02$ & $0.37 \pm 0.07$ & $50.86 \pm 9.87$ & $6.70 \pm 0.75$ \\
\hline $\mathrm{IL}-12(\mathrm{p} 40)$ & $178.46 \pm 13.74$ & $9.52 \pm 0.68$ & $0.83 \pm 0.04$ & $9.62+0.71$ & $7.38+0.57$ & $6.16 \pm 0.42$ & $23.62 \pm 1 .+1$ & $1.14 \pm 0.16$ & $133.43 \pm 17.68$ & $20.79 \pm 1.28$ \\
\hline IL-12 (p70) & $81.64 \pm 4.07$ & $4.37 \pm 0.22$ & $0.38 \pm 0.01$ & $4.36 \pm 0.16$ & $3.50 \pm 0.29$ & $2.81 \pm 0.09$ & $10.90 \pm 0.37$ & $0.54 \pm 0.07$ & $65.39 \pm 8.91$ & $9.50 \pm 0.15$ \\
\hline IL-13 & $43.33 \pm 2.29$ & $2.32+0,14$ & $0.20 \pm 0.01$ & $2.30 \pm 0.08$ & $1.85 \pm 0.15$ & $1.48 \pm 0.04$ & $5.79 \pm 0.26$ & $0.30 \pm 0.04$ & $34.54 \pm 4.88$ & $5.07 \pm 0.18$ \\
\hline IL-17 & $271.82+49.98$ & $14.12+2.26$ & $1.18 \pm 0.17$ & $13.79 \pm 2.17$ & $11.32 \pm 2.08$ & $8.61 \pm 1.16$ & $35.64 \pm 6.16$ & $1.62 \pm 0.35$ & $238.28 \pm 54.48$ & $29.94 \pm 4.64$ \\
\hline Eotaxin & $*$ & $0.06 \pm 0.01$ & $0.01 \pm 0.01$ & $0.06 \pm 0.01$ & $0.04 \pm 0.01$ & $0.04 \pm 0.01$ & $0.14 \pm 0.01$ & $0.01 \pm 0.01$ & $0.79 \pm 0.11$ & $0.12 \pm 0.01$ \\
\hline G-CSF & $*$ & $*$ & $0.09 \pm 0.01$ & $1.03 \pm 0.05$ & $0.82 \pm 0.07$ & $0.66 \pm 0.03$ & $2.56 \pm 0.11$ & $0.14 \pm 0.02$ & $14.78 \pm 1.98$ & $2.26 \pm 0.11$ \\
\hline GM-CSF & * & * & * & $11.47 \pm 0.48$ & $9.13 \pm 0.74$ & $7.36 \pm 0.26$ & $28.51 \pm 0.96$ & $1.41+0.18$ & $167.44 \pm 22.00$ & $24.94 \pm 0.58$ \\
\hline IFN- $\gamma$ & * & * & * & $*$ & $0.81 \pm 0.08$ & $0.66 \pm 0.06$ & $2.55 \pm(1) .11$ & $0.13 \pm 0.06$ & $15.31 \pm 0.42$ & $2.23 \pm 0.14$ \\
\hline $\mathrm{KC}$ & * & $*$ & $*$ & $*$ & $*$ & $0.87 \pm 0.06$ & $3.36 \pm 0.21$ & $0.18 \pm 0.03$ & $18.75 \pm 2.49$ & $3.00 \pm 0.22$ \\
\hline MCP-1 & * & * & * & $*$ & * & * & $3.92 \pm 0.16$ & $0.20 \pm 0.03$ & $23.04 \pm 3.07$ & $3.44 \pm 0.11$ \\
\hline MIP-1 $\alpha$ & * & * & * & * & $*$ & * & $*$ & $0.05 \pm 0.01$ & $5.74 \pm 0.70$ & $0.89 \pm 0.03$ \\
\hline MIP- $1 \beta$ & * & * & $*$ & * & $*$ & * & * & $*$ & $204.24 \pm 62.07$ & $25.29 \pm 5.36$ \\
\hline RANTES & $*$ & * & * & $*$ & $*$ & $*$ & * & $*$ & $*$ & $0.24 \pm 0.04$ \\
\hline
\end{tabular}

Shaded boxes highlight significantly different ratios from those reported in serum: light grey, $P<0.05$; mid-grey, $P<0.01$; dark grey, $P<0.001$. No Statistical analyses were

performed for IL-3 and RANTES due to their absent/poor detection levels in serum. 
in cytokine profile between serum and seminal fluid, and highlight which ones are likely candidates in matinginduced immunomodulation, the reported values may differ from those of older stud mice. In this respect it has been reported that male potency to elicit an immunomodulatory response in the female tract is linked to stud sexual status and maturity (Robertson et al. 1996b); older, more experienced males may thus exhibit slightly different cytokine profiles to those reported herein.

Although the array of cytokines investigated in the present study does not include all the potential participants in mating-induced immunomodulation (e.g. TGF- $\beta_{1}$ ), these findings nevertheless greatly extend our understanding of the immunoregulatory mediators of early pregnancy. The difficulty associated with investigating these factors in vivo relates to their dynamic interactions and often short-lived increases in concentration. However, our limited understanding of organ culture and immune/other somatic cell functions in culture also limits how representative in vitro models of cytokine-leukocyte networks are likely to be. Due to the inhibitory/synergistic modus operandi of many elements of the cytokine network, multiplex immunoassays will probably prove to be a useful tool in clarifying the mechanisms underlying many immunological phenomena including the establishment of pregnancy, pseudopregnancy (in rodents), miscarriage, pre-eclampsia and susceptibility to sexually transmitted diseases. In this respect, species-specific differences in the production site for seminal plasma cytokines will have to be taken into consideration.

\section{Acknowledgements}

The authors wish to thank Debra Evans for her assistance in sample collection.

\section{References}

Basu S, Aballa TC, Ferrell SM, Lynne CM \& Brackett NL 2004 Inflammatory cytokine concentrations are elevated in seminal plasma of men with spinal cord injuries. Journal of Andrology 25 250-254.

Cardoso E, Arregger A, Coumroglon M, Andrada EC \& Andrada JA 1993 Seminal plasma modulates lymphokine-activated killer cell activity in vitro. Minerva Endocrinologica 18 109-114.

Chu TM, Nocera MA, Flanders KC \& Kawinski E 1996 Localization of seminal plasma transforming growth factor-beta1 on human spermatozoa: an immunocytochemical study. Fertility and Sterility $66327-330$.

Daher S, de Arruda Geraldes Denardi K, Blotta MH, Mamoni RL, Reck AP, Camano L \& Mattar R 2004 Cytokines in recurrent pregnancy loss. Journal of Reproductive Immunology 62 151-157.

Denison FC, Grant VE, Calder AA \& Kelly RW 1999 Seminal plasma components stimulate interleukin-8 and interleukin-10 release. Molecular Human Reproduction 5 220-226.

Fossiez F, Banchereau J, Murray R, Van Kooten C, Garrone P \& Lebecque S 1998 Interleukin-17. International Reviews of Immunology 16 541-551.

Frenette G, Tremblay RR, Dube JY, Lazure C \& Lemay M 1998 High concentrations of the macrophage migration inhibitory factor in human seminal plasma and prostatic tissues. Archives of Andrology 41 185-193.
Fujisawa M, Fujioka H, Tatsumi N, Inaba Y, Okada H, Arakawa S \& Kamidono S 1998a Levels of interferon alpha and gamma in seminal plasma of normozoospermic, oligozoospermic, and azoospermic men. Archives of Andrology 40 211-214.

Fujisawa M, Kanzaki M, Okuda Y, Okada H, Arakawa S \& Kamidono S $1998 b$ Stem cell factor in human seminal plasma as a marker for spermatogenesis. Urology 51 460-463.

Gutsche S, von Wolff M, Strowitzki T \& Thaler CJ 2003 Seminal plasma induces mRNA expression of IL-1beta, IL- 6 and LIF in endometrial epithelial cells in vitro. Molecular Human Reproduction 9 785-791.

Huleihel M, Lunenfeld E, Horowitz S, Levy A, Potashnik G, Mazor M \& Glezerman M 1999 Expression of IL-12, IL-10, PGE2, sIL-2R and sIL-6R in seminal plasma of fertile and infertile men. Andrologia 31 283-288.

Hunt JS, Manning LS \& Wood GW 1984 Macrophages in murine uterus are immunosuppressive. Cellular Immunology 85 499-510.

Johansson M, Bromfield JJ, Jasper MJ \& Robertson SA 2004 Semen activates the female immune response during early pregnancy in mice. Immunology 112 290-300.

Kayisli UA, Mahutte NG \& Arici A 2002 Uterine chemokines in reproductive physiology and pathology. American Journal of Reproductive Immunology 47 213-221.

Kelly RW 1995 Immunosuppressive mechanisms in semen: implications for contraception. Human Reproduction 10 1686-1693.

Kelly RW 1999 Immunomodulators in human seminal plasma: a vital protection for spermatozoa in the presence of infection? International Journal of Andrology 22 2-12.

Kelly RW, Carr GG \& Critchley HO 1997 A cytokine switch induced by human seminal plasma: an immune modulation with implications for sexually transmitted disease. Human Reproduction 12 677-681.

Knudsen E, Iversen PO, Van Rooijen N \& Benestad HB 2002 Macrophage-dependent regulation of neutrophil mobilization and chemotaxis during development of sterile peritonitis in the rat. European Journal of Haematology 69 284-296.

Koch E \& Ellendorff F 1985 Detection of activity similar to that of early pregnancy factor after mating sows with a vasectomized boar. Journal of Reproduction and Fertility 74 39-46.

Liang ZG, Kamada M \& Koide SS 1993 Binding of a specific subclass of immunoglobulins by a human seminal plasma component. Andrologia 25 279-282.

Lin H, Mosmann TR, Guilbert L, Tuntipopipat S \& Wegmann TG 1993 Synthesis of T helper 2-type cytokines at the maternal-fetal interface. Journal of Immunology 151 4562-4573.

Maccioni M, Riera CM \& Rivero VE 2001 Identification of rat prostatic steroid binding protein (PSBP) as an immunosuppressive factor. Journal of Reproductive Immunology 50 133-149.

Maegawa $\mathbf{M}$, Kamada $\mathbf{M}$, Irahara $\mathbf{M}$, Yamamoto $\mathbf{S}$, Yoshikawa $\mathbf{S}$, Kasai Y, Ohmoto Y, Gima H, Thaler CJ \& Aono T 2002 A repertoire of cytokines in human seminal plasma. Journal of Reproductive Immunology 54 33-42.

Matalliotakis I, Kyriakou D, Fragouli Y, Loutradis D, Goumenou A \& Koumantakis E 1998a Determination of interleukin-11 in seminal plasma and elevated IL-11 in seminal plasma of infertile patients with urogenital infection. Archives of Andrology 41 177-183.

Matalliotakis I, Sifakis S, Goumemou A, Fragouli Y, Neonaki M, Matalliotakis G \& Koumantakis E 1998b Cytokine levels in seminal plasma. Clinical and Experimental Obstetrics and Gynecology 25 58-60.

Matalliotakis I, Arici A, Goumenou A, Koumantakis G, Selam B, Matalliotakis G \& Koumantakis E 2002 Distinct expression pattern of cytokines in semen of men with genital infection and oligoterato-asthenozoospermia. American Journal of Reproductive Immunology 48 170-175.

Miao D, Skibinski G \& James K 1996 The effects of human seminal plasma and PGE2 on mitogen induced proliferation and cytokine production of human splenic lymphocytes and peripheral blood 
mononuclear cells. Journal of Reproductive Immunology $\mathbf{3 0}$ 97-114.

Mould AW, Matthaei KI, Young IG \& Foster PS 1997 Relationship between interleukin-5 and eotaxin in regulating blood and tissue eosinophilia in mice. Journal of Clinical Investigation 99 1064-1071.

Naz RK \& Leslie MH 2000 Immunobiologic implication of RANTES in seminal plasma of fertile, infertile and immunoinfertile men. American Journal of Reproductive Immunology 44 197-204.

Naz RK \& Stanley ER 1995 Enhanced levels of colony stimulating factor-1 (CSF-1) in sera and seminal plasma of antisperm antibodypositive infertile men. Archives of Andrology 35 5-11.

Nocera M \& Chu TM 1995 Characterization of latent transforming growth factor-beta from human seminal plasma. American Journal of Reproductive Immunology 33 282-291.

O'Leary S, Jasper MJ, Warnes GM, Armstrong DT \& Robertson SA 2004 Seminal plasma regulates endometrial cytokine expression, leukocyte recruitment and embryo development in the pig. Reproduction 128 237-247.

Omu AE, al-Othman S, Mohamad AS, al-Kaluwby NM \& Fernandes S 1998 Antibiotic therapy for seminal infection. Effect on antioxidant activity and T-helper cytokines. Journal of Reproductive Medicine $43857-864$.

Orsi NM, Gopichandran N, Ekbote UV \& Walker JJ 2006 Murine serum cytokines throughout the estrous cycle, pregnancy and post partum period. Animal Reproduction Science [in press].

Paulis G, Conti E, Voliani S, Bertozzi MA, Sarteschi ML \& Fabris FM 2003 Evaluation of the cytokines in genital secretions of patients with chronic prostatitis. Archivio Italiano di Urologia, Andrologia 75 179-186.

Piccinni MP 2005 T cells in pregnancy. Chemical Immunology and Allergy 89 3-9.

Powell C, Orsi N, Simpson N \& Levene M 2004 Characterisation of the cytokine inflammatory response in LPS stimulated full-term cord blood. Journal of Perinatal Medicine 32 440-445.

Rees RC, Vallely P, Clegg A \& Potter CW 1986 Suppression of natural and activated human antitumour cytotoxicity by human seminal plasma. Clinical and Experimental Immunology 63 687-695.

Robertson SA 2005 Seminal plasma and male factor signalling in the female reproductive tract. Cell Tissue Research 2115909166.

Robertson SA \& Sharkey DJ 2001 The role of semen in induction of maternal immune tolerance to pregnancy. Seminars in Immunology 13 243-254.

Robertson SA, Mayrhofer G \& Seamark RF 1992 Uterine epithelial cells synthesize granulocyte-macrophage colony-stimulating factor and interleukin- 6 in pregnant and nonpregnant mice. Biology of Reproduction 46 1069-1079.

Robertson SA, Mayrhofer G \& Seamark RF 1996a Ovarian steroid hormones regulate granulocyte-macrophage colony-stimulating factor synthesis by uterine epithelial cells in the mouse. Biology of Reproduction 54 183-196.

Robertson SA, Mau VJ, Tremellen KP \& Seamark RF 1996b Role of high molecular weight seminal vesicle proteins in eliciting the uterine inflammatory response to semen in mice. Journal of Reproduction and Fertility 107 265-277.

Robertson SA, Mau VJ, Hudson SN \& Tremellen KP 1997 Cytokineleukocyte networks and the establishment of pregnancy. American Journal of Reproductive Immunology 37 438-442.

Robertson SA, O'Connell AC, Hudson SN \& Seamark RF 2000 Granulocyte-macrophage colony-stimulating factor (GM-CSF) targets myeloid leukocytes in the uterus during the post-mating inflammatory response in mice. Journal of Reproductive Immunology $\mathbf{4 6}$ $131-154$.

Robertson SA, Bromfield JJ \& Tremellen KP 2003 Seminal 'priming' for protection from pre-eclampsia - a unifying hypothesis. Journal of Reproductive Immunology 59 253-265.

Saxena S, Jha P \& Farooq A 1988 Purification and characterisation of an immunosuppressive factor from normal human seminal plasma. Journal of Reproductive Immunology 13 133-146.

Shimoya K, Matsuzaki N, Ida N, Okada T, Taniguchi T, Sawai K, Itoh S, Ohashi K, Saji F \& Tanizawa O 1995 Detection of monocyte chemotactic and activating factor (MCAF) and interleukin (IL)- 6 in human seminal plasma and effect of leukospermia on these cytokine levels. American Journal of Reproductive Immunology 34 311-316.

Strath M \& Sanderson CJ 1985 Production and functional properties of eosinophils from bone marrow cultures. Journal of Cell Science 74 207-217.

Sugita K, Hayakawa S, Karasaki-Suzuki M, Hagiwara H, Chishima F, Aleemuzaman S, Li JA, Nishinarita S \& Yamamoto T 2003 Granulocyte colony stimulation factor (G-CSF) suppresses interleukin (IL)-12 and/or IL-2 induced interferon (IFN)-gamma production and cytotoxicity of decidual mononuclear cells. American Journal of Reproductive Immunology 50 83-89.

Sun W, He X, Guo Z, Wang Q, Li X, Rayner J, Zhang L, Wang J \& Cao X 2004 IL-12p40-overexpressing immature dendritic cells induce $\mathrm{T}$ cell hyporesponsiveness in vitro but accelerate allograft rejection in vivo: role of NK cell activation and interferon-gamma production. Immunology Letters 94 191-199.

Tarter TH, Cunningham-Rundles S \& Koide SS 1986 Suppression of natural killer cell activity by human seminal plasma in vitro: identification of 19-OH-PGE as the suppressor factor. Journal of Immunology $1362862-2867$.

Tremellen KP, Seamark RF \& Robertson SA 1998 Seminal transforming growth factor beta1 stimulates granulocyte-macrophage colony-stimulating factor production and inflammatory cell recruitment in the murine uterus. Biology of Reproduction $\mathbf{5 8}$ $1217-1225$.

Vignali DA 2000 Multiplexed particle-based flow cytometric assays. Journal of Immunological Methods 243 243-255.

Wills-Karp M 1999 Immunologic basis of antigen-induced airway hyperresponsiveness. Annual Review of Immunology 17 255-281.

Wood GW, Hausmann E \& Choudhuri R 1997 Relative role of CSF-1, MCP-1/JE, and RANTES in macrophage recruitment during successful pregnancy. Molecular Reproduction and Development 46 62-70.

Xie ZF, Shi HZ, Qin XJ, Kang LF, Huang CP \& Chen YQ 2005 Effects of antigen presentation of eosinophils on lung Th1/Th2 imbalance. Chinese Medical Journal 118 6-11.

Zhang X, Xu H, Lin J, Qian Y \& Deng L 2005 Peritoneal fluid concentrations of interleukin-17 correlate with the severity of endometriosis and infertility of this disorder. British Journal of Obstetrics and Gynaecology 112 1153-1155.

Received 9 September 2005

First decision 17 November 2005

Revised manuscript received 18 November 2005

Accepted 28 November 2005 